\title{
Dopamine Modulates the Response of the Human Amygdala: A Study in Parkinson's Disease
}

\author{
Alessandro Tessitore, ${ }^{1}$ Ahmad R. Hariri, ${ }^{1}$ Francesco Fera, ${ }^{1}$ William G. Smith, ${ }^{1}$ Thomas N. Chase, ${ }^{2}$ \\ Thomas M. Hyde, ${ }^{1}$ Daniel R. Weinberger, ${ }^{1}$ and Venkata S. Mattay ${ }^{1}$ \\ ${ }^{1}$ Clinical Brain Disorders Branch, National Institute of Mental Health, and ${ }^{2}$ Experimental Therapeutics Branch, National \\ Institute of Neurological Disorders and Stroke, National Institutes of Health, Bethesda, Maryland 20892
}

In addition to classic motor signs and symptoms, Parkinson's disease (PD) is characterized by neuropsychological and emotional deficits, including a blunted emotional response. In the present study, we explored both the neural basis of abnormal emotional behavior in PD and the physiological effects of dopaminergic therapy on the response of the amygdala, a central structure in emotion processing. PD patients and matched normal controls (NCs) were studied with blood oxygenation level-dependent functional magnetic resonance imaging during a paradigm that involved perceptual processing of fearful stimuli. PD patients were studied twice, once during a relatively hypodopaminergic state (i.e., $\geq 12 \mathrm{hr}$ after their last dose of dopamimetic treatment) and again during a dopamine-replete state. The imaging data revealed a robust bilateral amygdala response in NCs that was absent in PD patients during the hypodopaminergic state. Dopamine repletion partially restored this response in PD patients. Our results demonstrate an abnormal amygdala response in PD that may underlie the emotional deficits accompanying the disease. Furthermore, consistent with findings in experimental animal paradigms, our results provide in vivo evidence of the role of dopamine in modulating the response of the amygdala to sensory information in human subjects.

Key words: Parkinson's disease; dopamine; amygdala; modulating emotions; BOLD fMRl; depression
There is converging evidence that the cardinal signs of Parkinson's disease (PD) (tremor, rigidity, and bradykinesia) are also accompanied by a progressive pattern of neuropsychological impairment (Dubois and Pillon, 1997) that includes abnormal emotion processing (Blonder et al., 1989; Borod et al., 1990; Pell, 1996; Benke et al., 1998; Breitenstein et al., 2001). Notably, patients with PD have deficits in the production of emotional responses and in the perception of both facial expressions (Jacobs et al., 1995; Breitenstein et al., 1998) and affective prosody (Crucian et al., 2001), behaviors that have been linked in part to the activity of dopaminergic neurons in the mesencephalon (Hori et al., 1993; Inglis and Moghaddam, 1999; Louilot and Besson, 2000; Greba et al., 2001; Rosenkranz and Grace, 2002). These findings suggest that the neuropathology of PD likely includes one or more components of the modular network subserving emotion processing. Although the hallmark of PD is dopaminergic neuronal loss in the substantia nigra pars compacta ( $\mathrm{SNpc}$ ), leading to striatal denervation and the classic motor signs and symptoms, there is also evidence of considerable degeneration of the nigromesolimbic dopaminergic system that originates in the ventral tegmental area (VTA) and medial SNpc (Javoy-Agid and Agid, 1980; Chinaglia et al., 1992). Importantly, the amygdala, a central structure in mediating emotional behavior, which receives dopaminergic inputs from the VTA and SNpc, has been implicated in the neuropathology of PD (Braak and Braak, 2000). Consistent with evidence from animal studies illustrating that sensory stimuli

\footnotetext{
Received May 20, 2002; revised July 29, 2002; accepted Aug. 6, 2002.

We thank Saumitra Das and Sam Lee for technical assistance.

Correspondence should be addressed to Dr. Venkata S. Mattay, Clinical Brain Disorders Branch, National Institute of Mental Health, 10 Center Drive, Room 3C108, Bethesda, MD 20892-1384. E-mail: vsm@helix.nih.gov.

Copyright (C) 2002 Society for Neuroscience 0270-6474/02/229099-05\$15.00/0
}

laden with affective value engage the amygdala, several recent neuroimaging studies have also revealed that facial expressions, especially those of negative effect, elicit a robust amygdala response in normal human subjects (Davidson and Irwin, 1999; Davis and Whalen, 2001).

In the current study, we explored the neural basis of abnormal emotional behavior in PD using blood oxygenation leveldependent (BOLD) functional magnetic resonance imaging (fMRI) to compare the response of the amygdala in patients with PD to that in age- and gender-matched healthy volunteers. We also sought to elucidate the physiological effects of dopaminergic manipulation on the response of the amygdala by studying patients in both relatively hypodopaminergic ("drug off") and dopamine (DA)-replete ("drug on") states. Toward these ends, we used an fMRI paradigm developed by Hariri et al. (2002), which has demonstrated a strong bilateral amygdala response during the perceptual processing of angry and fearful facial expressions.

\section{MATERIALS AND METHODS}

Subjects. Ten right-handed patients with idiopathic Parkinson's disease and 10 right-handed normal controls (NCs) gave written informed consent and participated in the study according to the guidelines of the National Institute of Mental Health Institutional Review Board. Before scanning, all patients underwent a detailed neurological examination. Two patients with Hoehn and Yahr stage I and eight with stage II (Hoehn and Yahr, 1967) (three females and seven males; mean age \pm SE, $59 \pm 3.2$ years) who were receiving dopaminergic pharmacotherapy (levodopa plus carbidopa alone or in combination with dopamine agonists) participated in the study. Five of them had a history of depression, and three of these five patients were taking antidepressant drugs (sertraline, paroxetine, and bupropion) at the time of our study. Each PD patient was studied twice in a counterbalanced manner during a drug-off state (i.e., $\geq 12 \mathrm{hr}$ after their last dopaminergic drug dose the night before) and during a drug-on state (i.e., 1-2 hr after the first dose of the day). Dopaminergic drug doses had been adjusted to provide an optimal 
A

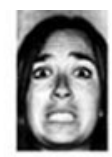

B

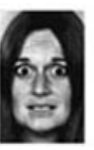

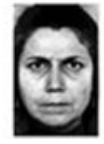

Figure 1. Experimental paradigm. A, During the emotion task, subjects viewed a trio of faces and had to select which one of two faces (bottom) expressed the same emotion as a target face (top). The identity of all three faces was always different, and an equal number of male and female faces was presented. $B$, During the control task, the subjects viewed a trio of geometric shapes and had to select which one of two shapes (bottom) was identical to a target shape (top).

therapeutic response. A brief neurological examination was performed before each scanning session to rate the severity of patients' motor function [Unified Parkinson's Disease Rating Scale (UPDRS) motor rating items 18-31] (Fahn and Elton, 1987).

Normal controls (three females and seven males; mean age \pm SE, $61 \pm$ 3.5 years) were matched for age, gender, and education and had no history of past or present neurological or psychiatric illness. All subjects were asked to refrain from nicotine and caffeine for $\geq 4 \mathrm{hr}$ before the fMRI studies.

Experimental paradigm. Both PD patients and NCs completed a blocked fMRI paradigm consisting of two experimental conditions. In the emotion task, subjects were required to select one of two facial expressions (either angry or afraid) that matched that of a simultaneously presented target expression (Fig. $1 A$ ). As a control task, subjects were required to match one of two geometric shapes with a simultaneously presented target shape (Fig. $1 B$ ).

There were five experimental blocks: two blocks of the emotion task interleaved with three control blocks, each lasting $32 \mathrm{sec}$. Before the beginning of each block, a brief instruction ("match emotion" or "match form") was presented for $2 \mathrm{sec}$. For the emotion task, 12 different images were used, six per block, three of each gender, all derived from a standard set of pictures of facial affect (Ekman and Friesen, 1976). Six different sets of geometric forms were used in the control condition. Images were presented for a period of $5 \mathrm{sec}$ and in a pseudo-randomized manner. During imaging, subjects responded with button presses, allowing for determination of accuracy and reaction time.

Data acquisition. BOLD fMRI data were collected, while subjects performed the task, on a General Electric 1.5T Signa scanner (Milwaukee, WI) using a gradient echo echoplanar imaging (EPI) sequence covering 24 axial, interleaved slices ( $4 \mathrm{~mm}$ thick, $1 \mathrm{~mm}$ gap), beginning at the cerebral vertex and encompassing the entire cerebrum and the majority of the cerebellum (repetition time/echo time, 2000/28 msec; field of view, $24 \mathrm{~cm}$; matrix, $64 \times 64)$. All scanning parameters were selected to optimize the quality of the BOLD signal while maintaining a sufficient number of slices to acquire whole-brain data. Before the collection of fMRI data for each subject, a reference EPI scan was acquired and carefully inspected for artifacts, such as susceptibility-related dropout in signal in the medial temporal lobes, particularly in the region of the amygdala, and ghosting. The fMRI data from all subjects for all scan sessions included in the analysis were devoid of such artifacts.

Data analysis. Image analysis was completed using SPM99 (www.fil. ion.ucl.ac.uk/spm). For each session and subject, the images were realigned to the first image in the time series to correct for head motion. These realigned images were then spatially normalized into a standard stereotactic space (Montreal Neurological Institute template) using a 12 parameter affine model. These spatially normalized images were smoothed to minimize noise and residual differences in gyral anatomy with a Gaussian filter, set at $8 \mathrm{~mm}$ full width at half maximum, producing an effective spatial resolution of $9.9 \times 9.9 \times 9.8 \mathrm{~mm}$. Data sets were then selected for their high quality as demonstrated by small motion correction $(<2 \mathrm{~mm})$ and matched voxel variance across sessions (Mattay et al., 1996). Based on these criteria, we excluded one PD patient from the final analysis, which was performed using nine PD patients and nine age/ gender-matched NCs. Voxel-wise signal intensities for each contrast image were ratio normalized to the whole-brain global mean.

These preprocessed data sets were analyzed using a second-level random effects model that accounts for both scan-to-scan and subject-tosubject variability (Holmes and Friston, 1998). This approach allows for a more critical and stringent exploration of BOLD responses than traditional fixed effects models that only account for scan-to-scan variability. To accomplish this second-level analysis, predetermined condition effects at each voxel were calculated using a $t$ statistic for each subject and session, producing a statistical image for the contrast emotion task versus control task. These individual contrast images were then used to identify both main effects of task for each group (PD drug off, PD drug on, and $\mathrm{NCs}$ ) using a one sample $t$ test as well as group differences in task-specific regional responses using either a paired $t$ test to determine differences in drug state within the PD population (drug off vs drug on) or an ANOVA to compare response differences between NCs and PD patients and between patients with a history of depression and those without, during both the drug-on and -off states.

A height and extent threshold of $p<0.05$ was used to identify significant voxels for all comparisons. A small volume correction for multiple comparisons was used for a priori regions of interest, namely the amygdala. We performed Student's $t$ test on clinical ratings (UPDRS, motor subscale scores) and task performance (percentage correct and reaction time) to determine significant differences across groups and drug states.

\section{RESULTS \\ BOLD fMRI results}

Consistent with previous reports (Davis and Whalen, 2001), perceptual processing of angry and fearful facial expressions was associated with a strong bilateral amygdala response in NCs (Fig. 2a) (Tables 1 and 2). Additional responses in NCs were identified in the bilateral posterior fusiform gyrus [Brodmann's area (BA) 19], ventral prefrontal cortex (PFC) (BA 47, 45), Broca's area (BA 44), anterior cingulate cortex (BA 32), and inferior occipital gyrus (BA 18/19).

In PD patients during the drug-off state, there was no significant amygdala response, but there were significant responses in the posterior fusiform gyri (Fig. 2b). However, there was a significant bilateral amygdala response in these same PD patients during the drug-on state (Fig. $2 c$ ). The responses of sensory and association cortices identified in NCs were also present in PD patients during both the drug-off and drug-on states (Fig. 2b,c).

These findings were also corroborated by direct comparisons between NCs and PD patients during both drug-off and drug-on states, which also highlighted interesting differences. Specifically, regions such as the amygdala and posterior fusiform gyrus showed a greater response in the NCs compared with both PD drug states (Fig. $3 a, b$ ). In contrast, a more robust Broca's area response was present in both PD drug states in comparison with NCs (Table 2). Within the PD patients, the direct comparison revealed an increase in the response of both the amygdala and right posterior fusiform gyrus in the drug-on state relative to the drug-off state (Fig. 3c). No brain regions exhibited a greater response in the drug-off state in comparison with the drug-on state. There was no significant difference in the response of the amygdala between patients with a history of depression and those without, during either the drug-on or -off state.

\section{Behavioral results}

All patients showed a significant improvement in their motor symptoms during the drug-on state when compared with the drug-off state (Table 3). There were no significant differences in either accuracy or reaction time for the control task across groups. Although the mean accuracy score for the emotion task was in general lower during the drug-off state compared with both the drug-on state and NCs, this difference did not reach statistical 
a) Normal Controls

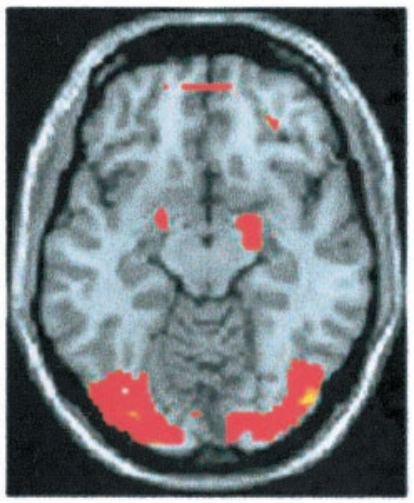

$Z=-14$ b) PD "drug-off"

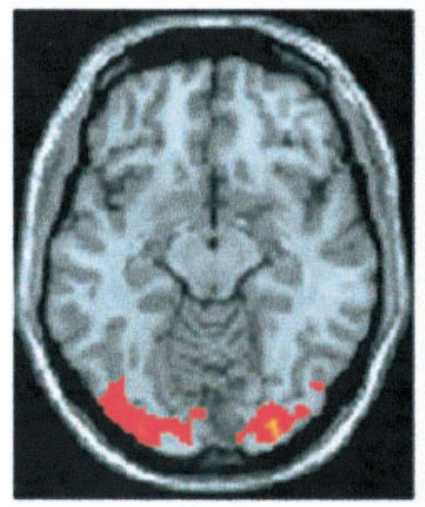

$Z=-12$ c) PD "drug-on"

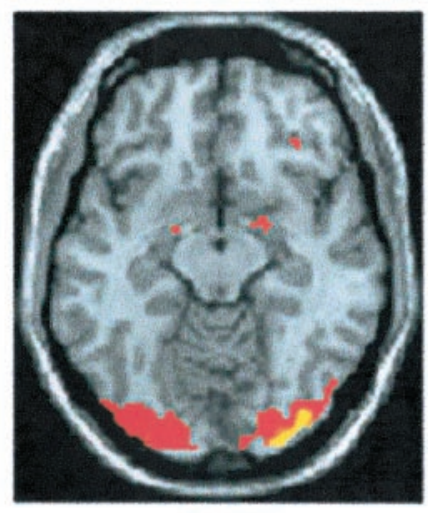

$Z=-12$

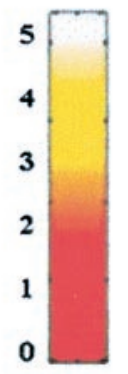

T value

Figure 2. Statistical parametric maps illustrating the difference in the BOLD response of the amygdala across the three groups $(p<0.05$, corrected). There was a robust bilateral amygdala response in NCs $(a)$ that was absent in patients during the drug-off state $(b)$. The same PD patients showed a significant amygdala response during the drug-on state $(c)$. Robust bilateral responses in the posterior fusiform gyrus are also represented in all three groups.

\begin{tabular}{|c|c|c|}
\hline Regions & Talairach coordinates $(x, y, z)$ & $Z$ score $^{a}$ \\
\hline \multicolumn{3}{|l|}{ Normal controls } \\
\hline Amygdala & $-/+14,-8,-12$ & $2.34 / 2.77$ \\
\hline Ventral prefrontal cortex & $-/+44,16,-7$ & $2.00 / 2.93$ \\
\hline Inferior frontal gyrus & $-/+37,6,28$ & $2.13 / 2.65$ \\
\hline Anterior cingulate cortex & $2,28,48$ & 2.72 \\
\hline Posterior fusiform gyrus & $-/+34,-56,-16$ & $2.30 / 3.04$ \\
\hline Inferior occipital gyrus & $-/+42,-82,-8$ & $2.98 / 3.24$ \\
\hline \multicolumn{3}{|l|}{ PD drug-off state } \\
\hline Amygdala & No significant response & \\
\hline Ventral prefrontal cortex & $+42,33,18 /-50,29,18$ & $2.09 / 2.64$ \\
\hline Inferior frontal gyrus & $-/+37,12,26$ & $2.68 / 2.00$ \\
\hline Anterior cingulate cortex & $0,26,42$ & 2.05 \\
\hline Posterior fusiform gyrus & $-/+34,-50,-16$ & $2.24 / 2.04$ \\
\hline Inferior occipital gyrus & $-/+42,-82,-8$ & $2.14 / 2.43$ \\
\hline \multicolumn{3}{|l|}{ PD drug-on state } \\
\hline Amygdala & $-16,-6,-12 /+14,-6,-12$ & $2.34 / 3.07$ \\
\hline Ventral prefrontal cortex & $-48,16,-5 /+40,23,-5$ & $2.05 / 2.64$ \\
\hline Inferior frontal gyrus & $-/+33,10,28$ & $2.84 / 2.23$ \\
\hline Anterior cingulate cortex & $0,26,42$ & 2.60 \\
\hline Posterior fusiform gyrus & $-/+36,-54,-12$ & $2.14 / 2.54$ \\
\hline Inferior occipital gyrus & $-/+42,-82,-8$ & $2.19 / 3.05$ \\
\hline
\end{tabular}

$\overline{\text { Coordinates and } Z \text { scores represent both left }(-\mathrm{x}) \text { and right }(+\mathrm{x}) \text { hemisphere }}$ responses.

${ }^{a} p<0.05$ corrected for multiple comparisons across a small volume of interest.

significance. There was no significant difference in reaction time for the emotion task across three groups.

\section{DISCUSSION}

Our results provide the first in vivo evidence of abnormal amygdala responses in patients with PD. In comparison with NCs, patients with PD in both the drug-off and drug-on states showed a reduced amygdala response during the perceptual processing of angry and fearful faces. Our results also provide the first in vivo demonstration in human subjects of dopamine modulation of the amygdala. Dopamine repletion appeared to partially restore the response of the amygdala in PD patients, as evidenced by the increased BOLD response in the drug-on state relative to the drug-off state.

The potentiated amygdala response in the dopamine-replete state relative to the hypodopaminergic state may reflect dopamine gating of amygdala inputs and subsequent increased amygdala neuronal activity. Recently, Hariri et al. (2002), using this same BOLD fMRI paradigm in healthy subjects, reported a robust potentiation of the response of the amygdala by dextroamphetamine, a nonspecific monoaminergic agonist that primarily acts via dopamine neurotransmission (Pierce and Kalivas, 1997; Zhang and Xu, 2001). Moreover, Rosenkranz and Grace $(1999,2001)$ via in vivo intracellular recordings, have shown that dopamine potentiates the response of the amygdala by attenuating the inhibitory influence of prefrontal inputs and augmenting the excitatory influence of sensory inputs. Thus, our findings appear to be consistent with their data.

In addition to the differential amygdala response, we observed a greater response of the fusiform gyri in NCs compared with PD patients, as well as in the dopamine-deplete relative to -replete states within PD. Several possible phenomena may underlie these observed differences. First, the amygdala, through excitatory feedback (Aggleton, 1993), can amplify the response in these object-specific processing regions in an effort to improve recognition and refine behavioral responses to affect-laden sensory stimuli (Morris et al., 1998). Thus, it is conceivable that the partial restoration of the response of these areas during the dopamine-replete state reflects the restoration of the amygdala response and subsequent positive feedback. Alternatively, the restoration of responses in these face-processing regions may reflect dopaminergic influences through direct midbrain projections. General deficits in face processing, in the absence of an affective component, have been reported in PD (Dewick et al., 1991). Finally, such response alterations may reflect more complex interactions between multiple interconnected brain regions and circuits. For example, the amygdala can drive the response of the PFC, through the locus ceruleus, and in turn, the PFC can drive the response of these sensory regions (LeDoux, 1997).

The observed differences in the physiological response of these brain regions between NCs and PD patients were clearly evident 
Table 2. Significant differences in BOLD responses between the three groups

Regions

Normal controls versus PD drug off

Amygdala

Posterior fusiform gyrus

Normal controls versus PD drug on

Amygdala

Posterior fusiform gyrus

PD drug off versus normal controls

Broca's area

PD drug on versus normal controls

Broca's area

PD drug on versus drug off

Amygdala

Posterior fusiform gyrus
Talairach coordinates $(x, y, z)$

$Z$ score $^{a}$

$\begin{array}{ll}-18,-6,-14 /+14,-8,-14 & 2.17 / 2.44 \\ -/+38,-58,-14 & 2.34 / 2.62 \\ & \\ +14,-8,-14 & 2.28 \\ -/+38,-58,-14 & 2.04 / 2.10 \\ -39,10,28 & 2.45 \\ -39,10,28 & \\ \end{array}$

$-16,-4,-12 /+14,-4,-12$

$1.91 / 2.06$

$+37,-83,-12$

Coordinates and $Z$ scores represent both left $(-x)$ and right $(+x)$ hemisphere responses.

${ }^{a} p<0.05$ corrected for multiple comparisons across a small volume of interest.
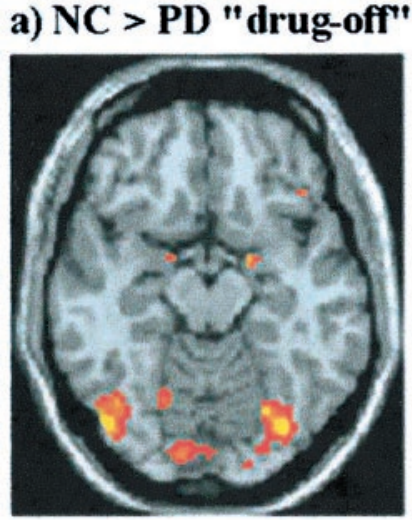

$Z=-14$
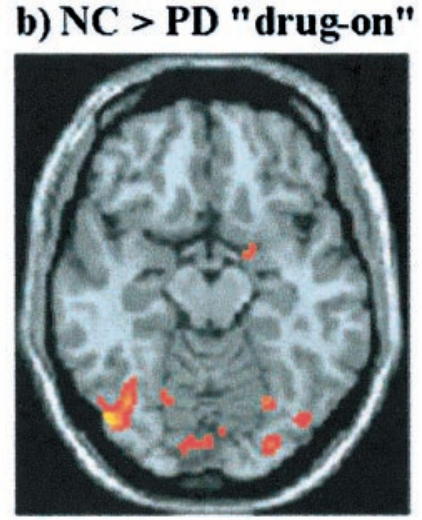

$Z=-14$ c) PD "drug-on" > "drug-off"
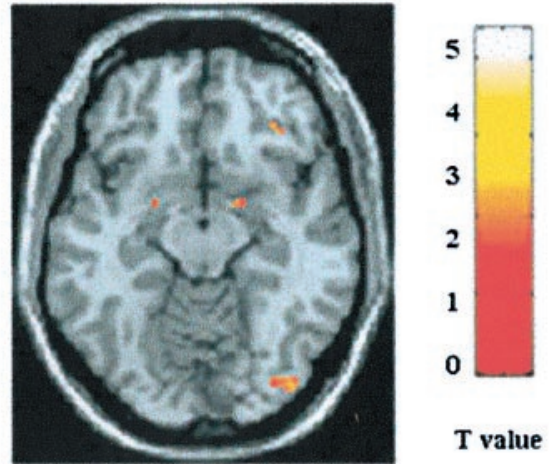

$Z=-12$

Figure 3. Statistical parametric maps illustrating results of direct comparisons between all three groups ( $p<0.05$, corrected). These comparisons revealed a greater BOLD response in the amygdala and posterior fusiform gyrus in NCs in comparison with PD patients during both drug-off state $(a)$ and drug-on state $(b)$. Dopamine repletion (PD drug on vs drug off) partially restored the response of the amygdala and right posterior fusiform gyrus (c).

Table 3. Clinical ratings, task accuracy, and reaction time (RT) measurements

\begin{tabular}{|c|c|c|c|c|c|}
\hline & $\begin{array}{l}\text { UPDRS (motor subscale) } \\
\text { mean } \pm \mathrm{SE}^{a}\end{array}$ & $\begin{array}{l}\text { Emotion }(\% \text { correct }) \\
\text { mean } \pm \mathrm{SE}\end{array}$ & $\begin{array}{l}\text { Emotion }(\mathrm{RT} \text { msec }) \\
\text { mean } \pm \mathrm{SE}\end{array}$ & $\begin{array}{l}\text { Control }(\% \text { correct }) \\
\text { mean } \pm \mathrm{SE}\end{array}$ & $\begin{array}{l}\text { Control (RT msec) } \\
\text { mean } \pm \text { SE }\end{array}$ \\
\hline Drug off state & $18 \pm 1.6$ & $81.2 \pm 5.06$ & $2646 \pm 73$ & $94.5 \pm 1.5$ & $1320 \pm 103$ \\
\hline Drug on state & $12 \pm 1.1$ & $87.5 \pm 3.9$ & $2351 \pm 71$ & $95.4 \pm 2.05$ & $1325 \pm 97$ \\
\hline Normal controls & & $88.8 \pm 3.3$ & $2285 \pm 83$ & $93.4 \pm 2.2$ & $1526 \pm 100$ \\
\hline
\end{tabular}

${ }^{a} p<0.001$

in the absence of significant differences at the level of observable behavior, with patients in both the hypodopaminergic and dopamine-replete states performing the task as well as NCs. The absence of a behavioral impairment in our patients, all in the early stages of the disease, is consistent with previous reports of normal recognition of facial emotions in mild to moderate PD (Adolphs et al., 1998; Breitenstein et al., 1998) and may reflect compensatory processes, such as the recruitment of canonical language regions (i.e., Broca's area) observed in the current study, to maintain task performance. Significant deficits in recognizing facial emotions, however, have been observed in subgroups of PD patients with severe symptomatology and cognitive impairment (Benke et al., 1998; Breitenstein et al., 1998). Thus, we speculate that advancing disease may decrease the efficacy of such compensatory mechanisms as patients develop degeneration of additional neuronal populations. Regardless of these potential long-term changes, our results suggest that the emotional deficits seen in PD may reflect the lack of engagement of the amygdala in response to affect-laden sensory stimuli and that dopamimetic treatment influences this response and, possibly, normal emotional behavior.

An abnormal functional response of the amygdala in patients with PD is consistent with previous reports demonstrating involvement of this region during the course of PD. Specifically, a $30-45 \%$ reduction of $2-\beta$-carbomethoxy-3 $\beta-\left(4-\left[{ }^{11} \mathrm{C}\right]\right.$ fluorophenyl $)$ tropane (phenyltropane analog of cocaine) binding has been reported not only in the striatum but also in the amygdala and the 
orbitofrontal cortex of patients with PD, even in the early stages of the disease, suggesting a loss of DA terminals (Ouchi et al., 1999). Moreover, postmortem studies have found severe neuropathological changes characterized by the presence of Lewy bodies in both input (accessory cortical nucleus) and output (central nucleus) regions of the amygdala in PD patients (Braak et al., 1994; Forno, 1996). Because of the influence of the amygdala on midbrain and hypothalamic centers regulating autonomic functions, such degenerative changes may contribute to the autonomic dysfunction (e.g., orthostatic hypotension and hyperhidrosis) frequently associated with PD (Chaudhuri, 2001).

Amygdala dysfunction has been implicated in the pathogenesis of depression (Drevets, 2001), an affective disorder linked to a variety of neurotransmitter abnormalities, including DA deficiency in mesocorticolimbic projections. Comorbid depression is also common in PD (Yamamoto, 2001) and may be related to the amygdala deficits we observe in our sample. In fact, five of the nine PD patients included in our analysis had a previous history of depression, and three of these five patients were taking antidepressant drugs at the time of our study. A post hoc analysis comparing subgroups, however, did not reveal any significant difference in the response of the amygdala during either the drug-on or -off states between these groups. Although comorbid depression in our small sample appears to be unrelated to differences in amygdala activity, such possible links merit additional exploration in larger samples of PD patients. Such investigations may prove important for understanding and treating the comorbid affective disturbances found in PD.

\section{REFERENCES}

Adolphs R, Schul R, Tranel D (1998) Intact recognition of facial emotion in Parkinson's disease. Neuropsychology 12:253-258.

Aggleton JP (1993) The contribution of the amygdala to normal and abnormal emotional states. Trends Neurosci 16:328-333.

Benke T, Bosch S, Andree B (1998) A study of emotional processing in Parkinson's disease. Brain Cogn 38:36-52.

Blonder LX, Gur RE, Gur RC (1989) The effects of right and left hemiparkinsonism on prosody. Brain Lang 36:193-207.

Borod JC, Welkowitz J, Alpert M, Brozgold AZ, Martin C, Peselow E, Diller L (1990) Parameters of emotional processing in neuropsychiatric disorders: conceptual issues and a battery of tests. J Commun Disord 23:247-271.

Braak H, Braak E (2000) Pathoanatomy of Parkinson's disease. J Neurol 247 [Suppl 2]:3-10.

Braak H, Braak E, Yilmazer D, de Vos RA, Jansen EN, Bohl J, Jellinger K (1994) Amygdala pathology in Parkinson's disease. Acta Neuropathol 88:493-500.

Breitenstein C, Daum I, Ackermann H (1998) Emotional processing following cortical and subcortical brain damage: contribution of the fronto-striatal circuitry. Behav Neurol 11:29-42.

Breitenstein C, Van Lancker D, Daum I, Waters CH (2001) Impaired perception of vocal emotions in Parkinson's disease: influence of speech time processing and executive functioning. Brain Cogn 45:277-314.

Chaudhuri KR (2001) Autonomic dysfunction in movement disorders. Curr Opin Neurol 14:505-511.

Chinaglia G, Alvarez FJ, Probst A, Palacios JM (1992) Mesostriatal and mesolimbic dopamine uptake binding sites are reduced in Parkinson's disease and progressive supranuclear palsy: a quantitative autoradiographic study using $\left[{ }^{3} \mathrm{H}\right]$ mazindol. Neuroscience 49:317-327.

Crucian GP, Huang L, Barrett AM, Schwartz RL, Cibula JE, Anderson JM, Triggs WJ, Bowers D, Friedman WA, Greer M, Heilman KM (2001) Emotional conversations in Parkinson's disease. Neurology $56: 159-165$.
Davidson RJ, Irwin W (1999) The functional neuroanatomy of emotion and affective style. Trends Cogn Sci 3:11-21.

Davis M, Whalen PJ (2001) The amygdala: vigilance and emotion. Mol Psychiatry 6:13-34.

Dewick HC, Hanley JR, Davies AD, Playfer J, Turnbull C (1991) Perception and memory for faces in Parkinson's disease. Neuropsychologia 29:785-802.

Drevets WC (2001) Neuroimaging and neuropathological studies of depression: implications for the cognitive-emotional features of mood disorders. Curr Opin Neurobiol 11:240-249.

Dubois B, Pillon B (1997) Cognitive deficits in Parkinson's disease. J Neurol 244:2-8.

Ekman P, Friesen WV (1976) Pictures of facial affect. Palo Alto, CA: Consulting Psychologists.

Fahn S, Elton RL (1987) Unified Parkinson's disease rating scale. In: Recent developments in Parkinson's disease (Fahn S, Marsden CD, Calne DB, Goldstein M, eds), pp 153-163. Florham Park, NJ: MacMillan Healthcare Information.

Forno LS (1996) Neuropathology of Parkinson's disease. J Neuropathol Exp Neurol 55:259-272.

Greba Q, Gifkins A, Kokkinidis L (2001) Inhibition of amygdaloid dopamine $\mathrm{D}(2)$ receptors impairs emotional learning measured with fearpotentiated startle. Brain Res 899:218-226.

Hariri AR, Mattay VS, Tessitore A, Fera F, Smith WG, Weinberger DR (2002) Dextroamphetamine potentiates the human amygdala response to fearful stimuli. Neuropsychopharmacology, in press.

Hoehn MW, Yahr MD (1967) Parkinsonism: onset, progression, and mortality. Neurology 17:427-445.

Holmes AP, Friston KJ (1998) Generalisability, random effects, and population inference. NeuroImage 7:S754.

Hori K, Tanaka J, Nomura M (1993) Effects of discrimination learning on the rat amygdala dopamine release: a microdialysis study. Brain Res 621:296-300.

Inglis FM, Moghaddam B (1999) Dopaminergic innervation of the amygdala is highly responsive to stress. J Neurochem 72:1088-1094.

Jacobs DH, Shuren J, Bowers D, Heilman KM (1995) Emotional facial imagery, perception, and expression in Parkinson's disease. Neurology 45:1696-1702.

Javoy-Agid F, Agid Y (1980) Is the mesocortical dopaminergic system involved in Parkinson disease? Neurology 30:1326-1330.

LeDoux J (1997) The emotional brain. New York: Touchstone.

Louilot A, Besson C (2000) Specificity of amygdalostriatal interactions in the involvement of mesencephalic dopaminergic neurons in affective perception. Neuroscience 96:73-82.

Mattay VS, Frank JA, Santha AK, Pekar JJ, Duyn JH, McLaughlin AC, Weinberger DR (1996) Whole-brain functional mapping with isotropic MR imaging. Radiology 201:399-404.

Morris JS, Friston KJ, Buchel C, Frith CD, Young AW, Calder AJ, Dolan RJ (1998) A neuromodulatory role for the human amygdala in processing emotional facial expressions. Brain 121:47-57.

Ouchi Y, Yoshikawa E, Okada H, Futatsubashi M, Sekine Y, Iyo M, Sakamoto M (1999) Alterations in binding site density of dopamine transporter in the striatum, orbitofrontal cortex, and amygdala in early Parkinson's disease: compartment analysis for beta-CFT binding with positron emission tomography. Ann Neurol 45:601-610.

Pell MD (1996) On the receptive prosodic loss in Parkinson's disease. Cortex 32:693-704.

Pierce RC, Kalivas PW (1997) A circuitry model of the expression of behavioral sensitization to amphetamine-like psychostimulants. Brain Res Brain Res Rev 25:192-216.

Rosenkranz JA, Grace AA (1999) Modulation of basolateral amygdala neuronal firing and afferent drive by dopamine receptor activation in vivo. J Neurosci 19:11027-11039.

Rosenkranz JA, Grace AA (2001) Dopamine attenuates prefrontal cortical suppression of sensory inputs to the basolateral amygdala of rats. J Neurosci 21:4090-4103.

Rosenkranz JA, Grace AA (2002) Dopamine-mediated modulation of odour-evoked amygdala potentials during pavlovian conditioning. Nature 417:282-287.

Yamamoto M (2001) Depression in Parkinson's disease: its prevalence, diagnosis, and neurochemical background. J Neurol 248 [Suppl 3]:5-11.

Zhang J, Xu M (2001) Toward a molecular understanding of psychostimulant actions using genetically engineered dopamine receptor knockout mice as model systems. J Addict Dis 20:7-18. 